\title{
Three Wide Binaries in LAMOST Pilot Survey
}

\author{
J. K. Zhao ${ }^{1}$, G. Zhao ${ }^{1}$ and T. D. Oswalt ${ }^{2}$ \\ ${ }^{1}$ Key Laboratory of Optical Astronomy, National Astronomical Observatories, Chinese \\ Academy of Sciences, Beijing 100012, China; email: zjk@bao.ac.cn, gzhao@bao.ac.cn \\ ${ }^{2}$ Physics and Space Science Department, Florida Institute of Technology, Melbourne, FL \\ 32901, USA email: toswalt@fit.edu
}

\begin{abstract}
Three wide binary candidates that have been identified in the Large sky Area MultiObject Spectroscopic Telescope (LAMOST) pilot survey data are presented. All three consist of main sequence stars of relatively late spectral type.
\end{abstract}

Wide binaries by definition have large semimajor axes (a $>100 \mathrm{AU}$ ). Each may be regarded as an open cluster with only two coeval components that have evolved independently, unaffected by such complications as mass exchange and tidal interactions that complicate the analysis of closer binaries. Such fragile pairs are important probes of the nature of halo dark matter, the evolution of the stellar halo, as well as the metallicities, masses, and ages of field stars.

Recent large-scale surveys such as the Sloan Digital Sky Survey, the Two Micron All Sky Survey, and the UKIRT Infrared Deep Sky Survey have yielded good photometric data that are useful in selecting more distant wide binaries when combined with proper motion information (Sesar et al. 2008; Dhital et al. 2010; Zhao et al. 2012). Using the catalog from the LAMOST pilot survey, correlated with proper motions from the PPMXL catalog, several new candidate pairs have been identified using the constraints of Dhital et al. (2010). Figure 1 displays the spectra of the first three wide binary candidates we have found. These three pairs all contain main sequence components of $\mathrm{F}, \mathrm{G}, \mathrm{K}$ or $\mathrm{M}$ spectral type. Radial velocity and metallicity measurements will be used to determine whether these and other wide binary candidates observed in the LAMOST survey are true physical pairs.
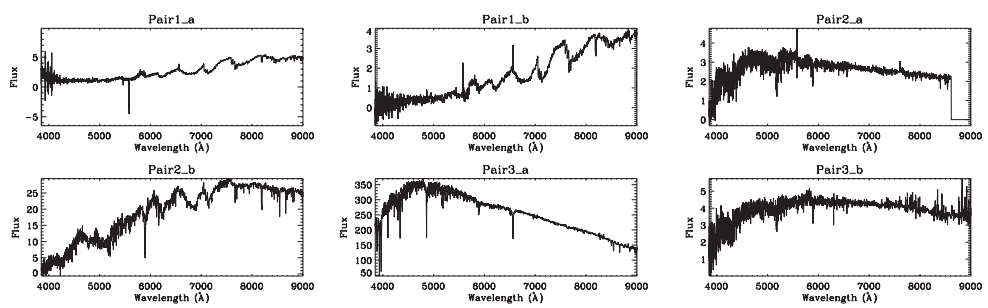

Figure 1. The spectra of the LAMOST wide binary candidates. Left to Right: LAMOST 1512+2952 (dM+dM); LAMOST 1306+2952 (dG+dK); LAMOST 1919+4059 (dF+dG).

\section{References}

Dhital, S., West, A. A., Stassun, K. G., \& Bochanski, J. J. 2010, AJ, 139, 2566

Sesar, B., Ivezić, Z., \& Jurić, M. 2008, ApJ, 689, 1244

Zhao, J. K., Oswalt, T. D., \& Zhao, G. 2012, AJ, 143, 31 